\title{
Application of the Platelet Rich Fibrin and L-PRF Leucocytes Technique in Long-Standing Periapical and Periodontal Lesion Defects
}

\section{Quevedo Bisonni M*, Sciaini Lewis V, Nuñez Otero M, Rodriguez K and Suárez JM}

Oral Surgery Department, Faculty of Medicine and Dentistry, University of Santiago de Compostela, Spain

*Corresponding Author: Quevedo Bisonni M, Oral Surgery Department, Faculty of Medicine and Dentistry, University of Santiago de Compostela, Spain.
Received: July 27, 2021

Published: August 26, 2021

(C) All rights are reserved by Quevedo Bisonni M., et al.

\begin{abstract}
Long-standing periapical and periodontal lesions present a common biological sequence, progressive resorption and loss of alveolar bone around the affected tooth. In recent years there has been an enormous evolution in the key role that platelets play in tissue regeneration techniques, facilitating, among other actions, the healing of soft tissues and hard tissues thanks to the release of cytokines and growth factors over an extended period of time. Platelets rich in fibrin and leukocytes is a second generation platelet concentrate, which we obtain without additives directly from the patient's blood with the aim of establishing a fibrin mesh that serves as an anchor to all the substances involved in the tissue regeneration process.
\end{abstract}

Keywords: Platelet Rich Fibrin; L-PRF Leucocytes Technique; Periodontal Lesion Defects

\section{Introduction}

The concept of regeneration in Dentistry can be defined as the reproduction or reconstruction of the lost part of a structure or anatomical system, with the aim of recovering and restoring its architecture and function. Numerous investigations over the years have had the main objective of trying to understand the phenomena that accompany the destruction of tissues, their recovery and healing, through the use of platelet concentrates [1].

Platelet concentrates must be understood as autologous biomaterials, obtained through cytapheresis, with the main objective of separating platelets.

In the last two decades,it has been better understood its physiological properties and its importance in the wound healing process. which has multiplied its indications and therapeutic applications, especially in the field of oral surgery and implantology [2].

The autologous platelet concentrates that we use as a basis for treatment in regenerative procedures, consisting in a fraction of plasma with a platelet content higher than usual and characterized by the presence of three components: platelets, leukocytes and fibrin $[3,4]$.

The scientific basis for its clinical use is established in the initial phases of healing - inflammatory phases - in which the release of platelet granules occurs with the consequent formation of a clot, the release of chemotactic and pro-inflammatory molecules and a wide variety of growth factors: platelet-derived growth factor (PDGF), transforming growth factor (TGF), platelet factor 4 (PF4), platelet-derived angiogenic factor (PDAF), endothelial growth 
factor vascular (VEGF) and epidermal growth factor (EGF), which are capable of stimulating the differentiation of mesenchymal tissue and initiating tissue repair processes [5].

Even today, there is great confusion with the terms that are reflected in the different classifications. One of the most didactic is the one carried out by Dohan Ehrenfest in 2009 [4,5], which divided platelet derivatives into four groups depending on their leukocyte content and fibrin architecture: pure platelet-rich plasma, platelet and leukocyte rich plasma, pure platelet rich fibrin, and platelet and leukocyte rich fibrin.

Pure Platelet Rich Plasma (P-PRP) and Platelet Rich Plasma and Leukocytes (L-PRP). These are liquid platelet suspensions, with and without leukocytes that are used as injectable suspensions. After their activation with thrombin, calcium chloride, batroxobin and other agents, they are transformed into fibrin gel with a certain architecture [5].

Pure Platelet Rich Fibrin (P-PRF) and Platelet and Leukocyte Rich Fibrin (L-PRF). They are solid fibrin biomaterials,with and without leukocytes. It can be natural (L-PRF) or artificial (P-PRF), but in both techniques, the subsequent activation of platelets occurs without the addition of activating substances, generating a strong structured fibrin matrix $[6,7]$.

It was Dr. Choukroun, an anesthesiologist dedicated to the treatment of chronic pain, who first proposed the use of PRF, for the healing process of long-standing torpid ulcers and for treatment of their painful manifestations. In 2006 he proposed the use of PRF in different dental treatments [4].

Currently, the use of platelet-rich fibrin and leukocytes, instead of platelet-rich plasma, is based on the ability of the former to generate a much more stable and firm clot, which facilitates its manipulation in the area of the defective tissue, maintaining biological factors like PRP, but in a higher proportion, obtained by a stricter protocol, faster handling and collection, prolonged permanence in vivo, while facilitating a clear reduction in costs $[5,6]$.

This technique (L-PRF) has a clear indication in implantology and oral surgery, especially in complementary regeneration techniques. It has been used with excellent clinical results in sinus lift techniques, in which it has been found that it can significantly stimulate bone formation and tissue angiogenesis in Schneiderian membrane perforations $[8,9]$.

Likewise, for its use in bone regeneration techniques in oral surgery, the action of the well-known RUNX2 protein is essential, considered a key transcription factor linked to the differentiation process of osteoblasts. Today we know that the progressive release of these proteins, which are found in the fibrin matrix, has, among other functions, that of promoting cell migration of structures such as the periodontal ligament and gingival fibroblasts [10].

The use of this technique in post-extraction sockets has also shown bone formation six weeks after its use and without signs of bone resorption. As occurs in the clinical case that we present, when one or more walls are absent or partially reabsorbed, the combined use of L-PRF and bone substitutes is recommended, demonstrating its great capacity to act as a biological connector between bone particles. Similarly, it has been possible to verify a high efficacy on the control of pain and postoperative edema [11].

All the components that make up this biomaterial are found in the patient's body, and by not incorporating additives, it is transformed via an almost physiological process, which concentrates the different elements in the area that we wish to regenerate [12].

A large part of the mechanisms involved in tissue regeneration are based on the action of multiple proteins, which make up an authentic cocktail of bioactive molecules, including adhesive proteins, cytokines, chemokines, coagulation factors, antimicrobial proteins, glycoproteins (fibronectin and vitronectin, especially active during the first week after use), proteases and antiproteases, chondroitin, albumin, immunoglobulins and other essential proteins such as platelet factor 4 , thromboglobulin and endostatins $[13,14]$.

On the other hand, leukocytes, responsible for multiple functions, respond to the presence of integrins, which are glycoproteins that actively participate in the adhesion of leukocytes to the vascular endothelium. These integrins also have a close link with fibrin, as they are formed thanks to the expression by fibrin of CD 11c receptors that bind to CD 18. This CD 11c/CD 18 union is what facilitates the processes of migration and activation of other leukocytes with neutrophils [15].

Citation: Quevedo Bisonni M., et al. "Application of the Platelet Rich Fibrin and L-PRF Leucocytes Technique in Long-Standing Periapical and Periodontal Lesion Defects". Acta Scientific Dental Sciences 5.9 (2021): 143-151. 
The activity of leukocytes has several target functions of interest: immune regulation, which intervenes in the production of multiple cytokines with pro-inflammatory activity, such as interleukin IL and IL-6, also IL-4 synthesized by Th lymphocytes, mast cells and basophils.

All these processes that we have just described occur in the repair area, simultaneously with the production of growth factors, such as the transforming growth factor derived from platelets, the fibroblast growth factor, with which it is possible to stimulate fibroblasts and induce collagen production, a process that in turn will promote angiogenesis. In this way and as a consequence, the blocking of bacterial proteases will be effective, with the consequent antibacterial effect [16].

As in the clinical case that we are going to describe below, the processes of bone regeneration after a chronic periodontal injury or a loss of several alveolar walls due to the presence of a previous apical granuloma or a cyst, is one of the current therapeutic challenges. For this reason it is recommended, platelets rich fibrin and leukocytes.

\section{Clinical Case}

54-year-old woman, who attends the Oral Surgery Teaching Unit of the Faculty of Dentistry and Medicine of the University of Santiago de Compostela, presenting a long-standing endoperiodontal lesion, with complete loss of the vestibular wall, reabsorption of the inserted gingiva, and exposure of a large part of the root and grade II tooth mobility (Figure 1-3).

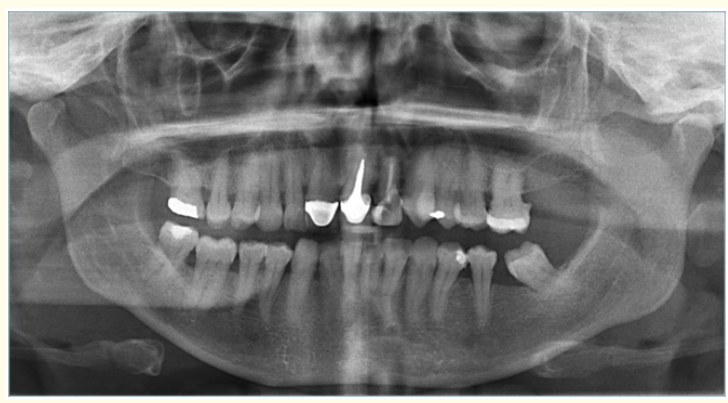

Figure 1: Previous state of the patient.

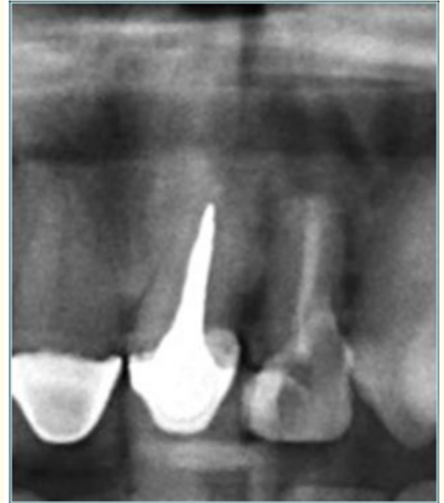

Figure 2: Endoperiodontal injury in 22.

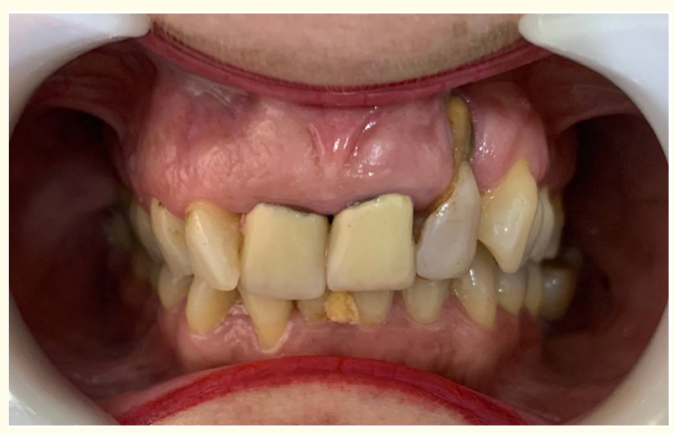

Figure 3: Exposure and resorption of tooth 22 root.

The treatment plan sequence was exodontia of the tooth 22 , thorough debridment of the remaining granulation tissue, placement particulate bone and a pericardium membrane in the alveolus. Before replacing the mucoperiosteal flap, we performed a Rehrmann [19], making an incision in the periosteum at the base of the flap, to expand its surface, until the residual cavity was sealed.

After six months healing period, in which the patient was wearing a provisional fixed prosthesis, we decided to use the conventional surgical technique with plaltelets rich in fibrin and leukocytes (L-PRF) to induce regeneration and provide the patient with an adequate emergence profile obtaining Platelet Rich Fibrin and leukocytes L-PRF. 
Necessary material

To carry out the L-PRF technique we need a centrifuge that has, among other adjustable parameters, time and revolutions. To perform the venipuncture, we have a nursing graduate in our unit who uses a conventional extraction kit to puncture the patient. We also need tissue regeneration material and conventional oral surgery material.

Blood is taken from the antecubital vein of the patient in $9 \mathrm{ml}$ tubes and it is immediately centrifuged, in this case in the IntraSpin centrifuge, at 2,700 rpm for 12 minutes [20] (18 $\mathrm{min}$ in the case of anticoagulated patients). Each red tube is for a fibrin plug or membrane and we need at least 1 white tube for fibrinogen, to be used in the preparation of the L-PRF block. The arrangement of the tubes in the centrifuge drum must be even in number so that it is balanced and allows the correct centrifugation process.

The tubes that we use for blood collection must be approved for clinical use according to the ISO 10993 standard, since the tubes that we commonly use for other purposes can induce cytotoxicity, mutagenicity, intradermal irritation and hemolysis, among many other undesirable effects [5].

Immediately after its extraction, the blood begins to coagulate when it comes into contact with the walls of the tube, so that the fibrinogen is initially concentrated in the middle - upper part of the tube and later the circulating thrombin will transform it into fibrin, creating a clot located in the medial part of the tube after centrifugation [5].

Erythrocytes are located in the lower part, while acellular plasma is in the upper part of the tube. The portion of the sample that we collect is the fibrin and platelet clot, once the erythrocyterich layer has been separated.

Next, with the Xpression box we are going to create membranes of constant thickness, we deposit the fibrin clot in the box, cover it with the pressing tray and in turn cover it with the lid, with this technique we guarantee its hydration for at least five hours.

Simultaneously we cut the pericardium membrane with a scalpel and hydrate it with saline solution, before placing the membrane in the bone defect (Figure 4).

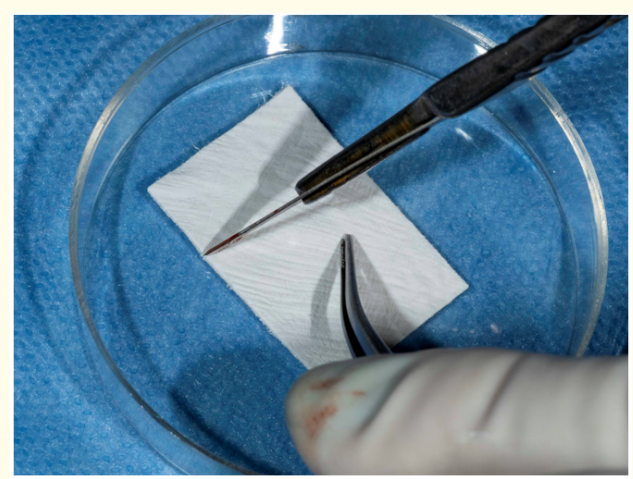

Figure 4: We cut and configure the pericardial membrane and hydrate with saline solution.

We raised a full-thickness buccal and palatal flap in the area of the tooth 22, and we adjust the size of the membrane to the bone defect, and we fixed the membrane to the vestibular bone wall using a fixation screw (Figure 5-7).

Next, we prepare the L-PRF Block: two membranes are cut into very small parts and mixed with particulate bone in a container in a proportion of $2 \mathrm{~L}$-PRF membranes for each $0.5 \mathrm{~g}$ of particulate bone, hydrating the mixture with L-PRF exudate of the Xpression Kit. This mixture is incorporated on a fibrinogen base and then more fibrinogen is added on top of the mixture and we leave it enough time until $\mathrm{f}$ it agglutinates, trying to give it the approximate shape of the defect that we wish to regenerate (Figure 8). After a few minutes, the L-PRF block will have sufficient elasticity and consistency to be transferred and to be adapted to the surgical area.

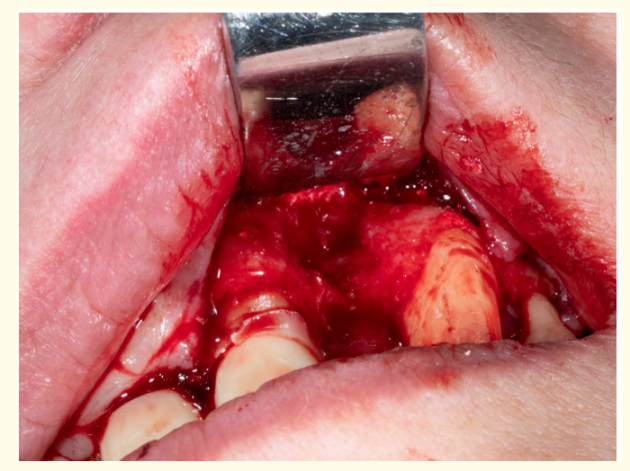

Figure 5: We lift a full thickness mucoperiosteal flap. 


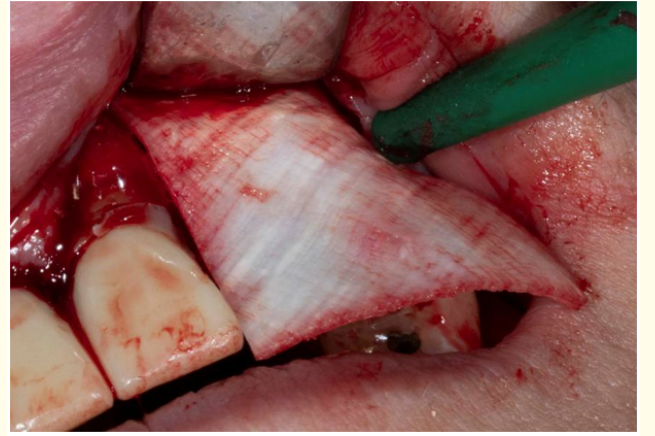

Figure 6: We test the spatial adaptation of the membrane.

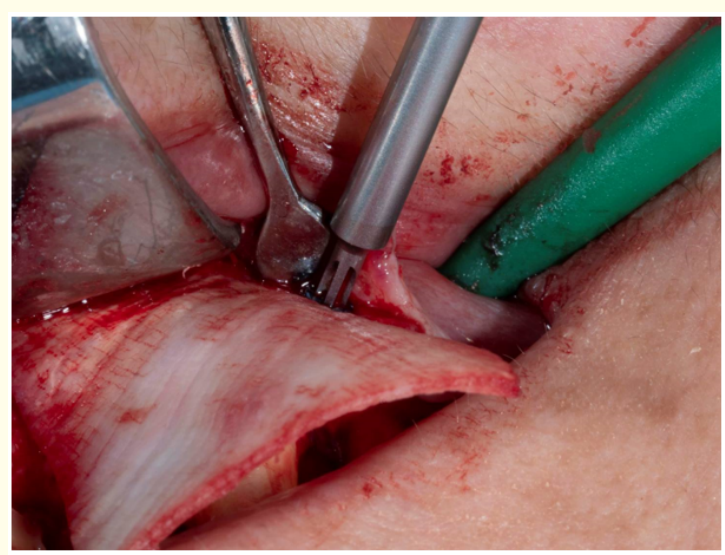

Figure 7: We fix the membrane with a microscrew.

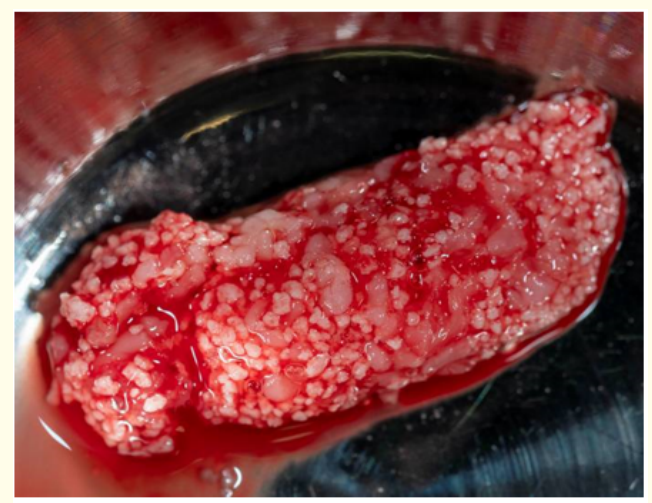

Figure 8: The PRF - Block must be elastic and moldable.
With the help of a biomaterial condenser, we adapt the L-PRF block to the bone defect, so that the gap in the residual cavity is as small as possible (Figure 9).

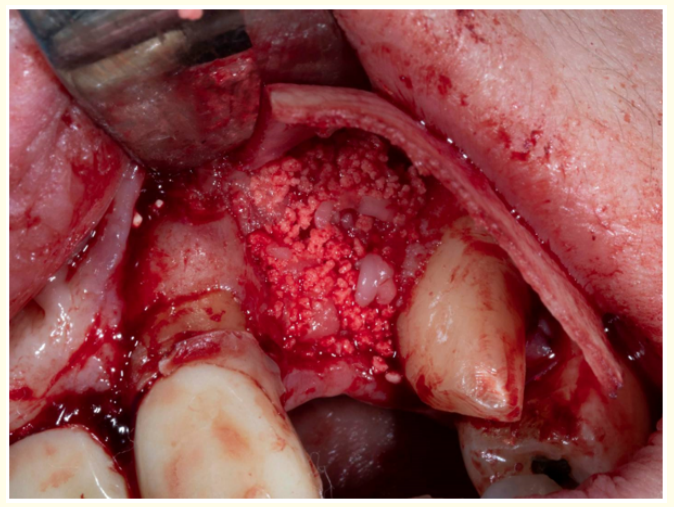

Figure 9: The material must completely fill the cavity.

We fixed the pericardium membrane palatally using a fixation screw permanently adapted it to the entire surface of the defect (Figure 10). Subsequently, we placed two L-PRF membranes that we had preserved in the Xpression Kit on top, in such a way that when overlapping and adapting them, the pericardium membrane was completely covered.

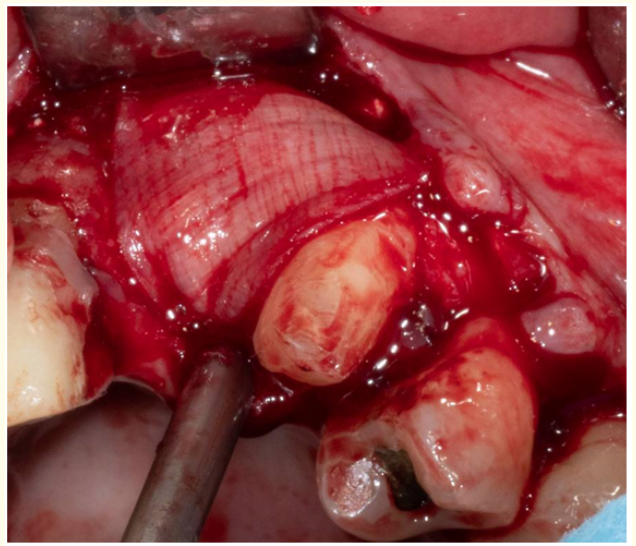

Figure 10: We fix the membrane by palatal with a microscrew and we adapt to the entire surface. 
The closest part to the erythrocyte layer is oriented towards the area to be regenerated, since it is the one that contains more growth factors, because the platelets are not distributed equally within and on the surface of the L-PRF clot (Figure 11-13).

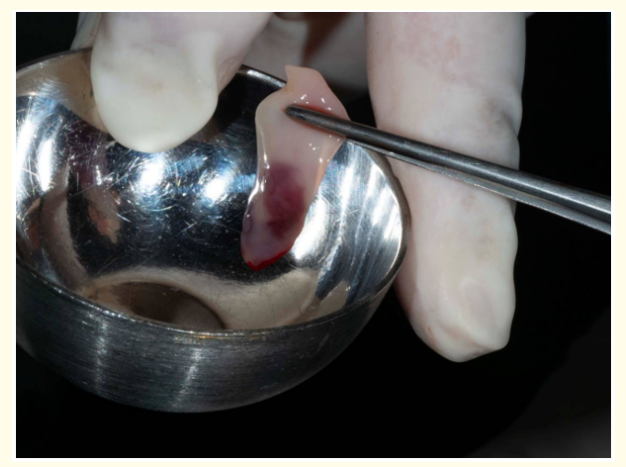

Figure 11: We transport the autologous membranes that we had preserved.

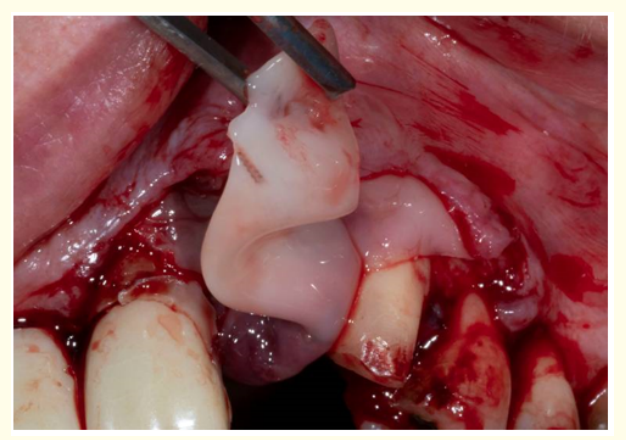

Figure 12: We progressively adapt one of the membranes.

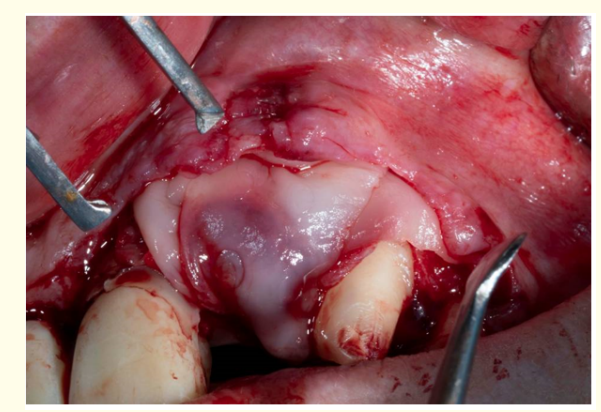

Figure 13: The membranes must fill the defect.
Finally, we replace the mucoperiosteal flap and suture with Supramid 4/0, placing loose stitches, with the least possible tension, and the excess exudate is injected into the surgical area. After the intervention, a fixed resin provisional previously made, is placed without pressure over the tissues (Figure 14-16).

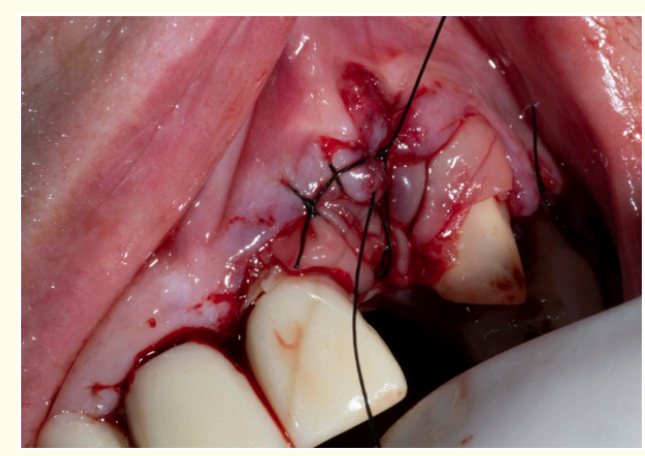

Figure 14: Suture with simple points 4.0.

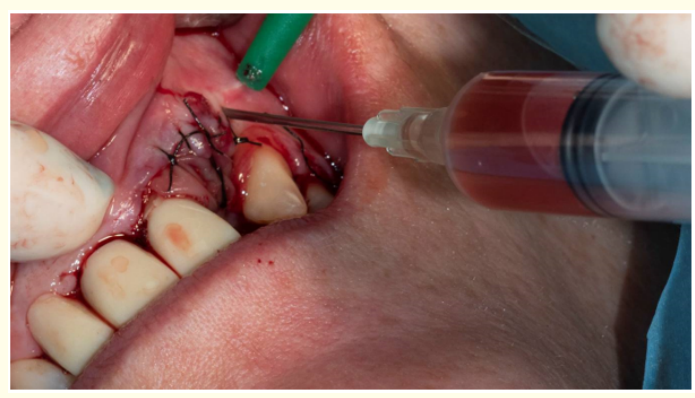

Figure 15: We hydrate with the excess exudate.

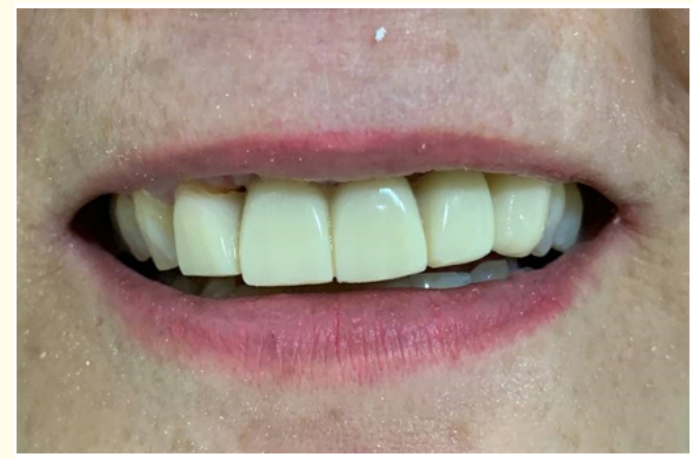

Figure 16: Provisional placement. 


\section{Discussion}

Since Choukroun., et al. described the first platelet- and leukocyte-rich concentrate in a fibrin matrix [21] the interest generated by platelet concentrates has increased. Compared with PRP, the L-PRF has a higher quantity of platelets and leukocytes, as well as growth factors such as PDGF, VEGF and TGF, and also has very representative proportions of fibrin, fibronectin and vitronectin $[14,22]$.

The high concentrations of thrombin present in the PRP form bilateral junctions that determine a mesh with a very rigid structure. However, the low thrombin concentration of L-PRF determines a more flexible structure capable of promoting cytokine entrapment and the migration of cells such as leukocytes, which contain VEGF. Its spatial arrangement serves as a substrate for platelets to chemotactically attract circulating stem cells [14,22]. While PRP is used as a layer of fibrin added to the surgical site, the strong and elastic architecture of the fibrin membrane of L-PRF allows its application as a true membrane or tissue, suturable, and with wide clinical versatility [23].

L-PRF has an ability to release growth factors and membrane proteins for more than 7 days far exceeding the 4 hours in which the PRP matrix disappears and its rapid release of growth factors. These advantages of "biological boosting" are obtained after a standardized preparation protocol, with a 100\% autologous biomaterial, a fast ( $<20 \mathrm{~min}$ ) and economical technique, facilitating its clinical incorporation with respect to the diversity of protocols for obtaining PRP, with slow, complex technique, several phases, use of anticoagulants and higher cost [22,24].

The use of these materials in dentistry is mainly based on the high tissue acceptance as they are autogenous components. Studies show that adding fibrinogen to bone substitute biomaterials, both allogenic and xenogenic, can influence osteoblastic activity in vivo. At the molecular level, they support a better tissue response, both at the level of soft and hard tissues, in the face of a greater presence of their own molecular families [25]. Some studies suggest that not only the response of the tissues is more satisfactory, but they also show that the presence of these agents could alleviate the postoperative symptoms inherent to any surgery [26], whether curative, regenerative or even palliative in severe cases such as communications due to osteonecrosis of the jaws $[27,28]$.
Combined injuries in dentistry have always been a great challenge, due to the great bacterial involvement, the complexity of performing the treatments, and the appearance of large residual defects. A recent study published this year in the Journal of Endodonthics shows how PRP improves osteo-odontogenic differentiation in stem cells from the apical papilla of immature third molars, promoting their maturation and migration, so its field of application could be greater [29]. Comparative studies of the technique with a natural healing process support the benefit of using L-PRF as a cavity filling material to achieve the preservation of the horizontal and vertical ridge dimension three months after tooth extraction [30].

In 2019, de Angelis published a study on a total of 45 patients, in which he performed three alveolar preservation techniques: application of L-PRF, application of L-PRF with xenograft and single application of xenograft. His results were significantly better when L-PRF and xenograft were combined [31].

It should be taken into account that healing in the oral cavity is compromised by multiple factors such as: high bacterial concentration, continuous contamination by degradation of molecules from food, terminal blood supply, local involvement of systemic diseases, etc. The limited evidence on the effects of L-PRF in intraoral bone graft procedures highlights the need for more research to fully evaluate its clinical indications [32]. We know that leukocytes, specifically lymphocytes, play a fundamental role in healing. Vehiculizing them through plasma provides us with a large amount of blood components, through which it is possible to alleviate the most acute postoperative phase, promote revascularization and improve tissue regeneration [33].

The use of these techniques is strongly supported at the microbiological level, but the risk of bias in clinical studies is high [34]. A greater number of studies with sufficient control cases are necessary to be able to identify the existing biases, mainly due to the heterogeneity of the methodologies used and the multifactoriality of the healing of the socket, to reduce its effect on sensitivity.

\section{Conclusion}

The clinical protocol for the application of Platelet Rich Fibrin and L-PRF leukocytes is a simple and predictable surgical procedure that uses biological elements from the patient himself,

Citation: Quevedo Bisonni M., et al. "Application of the Platelet Rich Fibrin and L-PRF Leucocytes Technique in Long-Standing Periapical and Periodontal Lesion Defects". Acta Scientific Dental Sciences 5.9 (2021): 143-151. 
thus minimizing the complications of other biomaterials, and makes a large amount of material available to us. biological to be used in tissue regeneration techniques applied to periapical and periodontal defects of the maxilla.

It is evident that a greater number of clinical trials are required in the medium and long term to verify its stability and evolution.

\section{Bibliography}

1. Baiju RM., et al. "Autologous platelet-rich fibrin: A boon to periodontal regeneration. Report of two different clinical applications". Health Sciences (2013): 1-13.

2. Gupta V., et al. "Regenerative potential of platelet rich fibrin in dentistry: Literature review". The Asian Journal of Oral Health and Allied Sciences 1 (2011): 22-28.

3. Caballero A., et al. "Bone regeneration of the alveolar ridge with platelet-rich fibrin before implant placement. A clinical case". Spanish Journal of Implants (2017).

4. Dohan Ehrenfest DM., et al. "Classification of platelet concentrates: from pure platelet-rich plasma (P-PRP) to leucocyteand platelet-rich fibrin (L-PRF)". Trends in Biotechnology 27 (2009): 158-167.

5. Salgado A., et al. "New trends in tissue regeneration: fibrin rich in platelets and leukocytes”. Revista Española de Cirugía Oral y Maxilofacial 39 (2017): 91-98.

6. Madurantakam P., et al. "Characterization of Leukocyte-platelet rich fibrin. A novel biomaterial". Journal of Visualized EXperiments 29 (2015): 10.

7. Preeja $\mathrm{C}$ and Arun S. "Platelet-rich fibrin: Its role in periodontal regeneration". The Saudi Journal for Dental Research 5 (2014): 117-122.

8. Jeong SM., et al. "Simultaneous sinus lift and implantation using platelet- rich fibrin as sole grafting material". Journal of Cranio-Maxillofacial Surgery 42 (2014): 990-994.

9. Oncu E and Kaymaz E. "Assessment of the effectiveness of platelet rich fibrin in the treatment of Schneiderian membrane perforation". Clinical Implant Dentistry and Related Research (2017): 1-6.

10. Xuan F., et al. "A comparative study of the regenerative effect of sinus bone grafting with platelet-rich fibrin-mixed Bio-Oss ${ }^{\circledR}$ and commercial fibrin-mixed Bio-Oss $囚$ : an experimental study". Journal of Cranio-Maxillofacial Surgery 42.4 (2014): e47-50.
11. Ozgul O., et al. "Efficacy of platelet rich fibrin in the reduction of the pain and swelling after impacted third molar surgery: Randomized multicenter split-mouth clinical trial". Head and Face Medicine 11 (2015): 37.

12. Leigha R. "Potential of platelet rich fibrin in regenerative periodontal therapy: literature review". Canadian Journal of Dental Hygiene 47.1 (2013): 33-37.

13. Anitua E., et al. "Infiltration of plasma rich in growth factors enhances in vivo angiogenesis and improves reperfusion and tissue remodeling after severe hind limb ischemia". Journal of Controlled Release 202 (2015): 31-39.

14. McLellan J and Plevin S. "Temporal release of growth factors from platelet-rich fibrin (PRF) and platelet-rich plasma (PRP) in the horse: A comparative in vitro analysis". The International Journal of Applied Research in Veterinary Medicine 12 (2014): 44-53.

15. Arce MA., et al. "Fibrin rich in platelets and leukocytes: Excellent autologous biomaterial for tissue regeneration (2018).

16. Moreno Díez R., et al. "Techniques for obtaining platelet-rich plasma and its use in osteoinductive therapy". Farmacia Hospitalaria 39.3 (2015): 130-136.

17. Chenchev IL., et al. "Application of platelet-rich fibrin and injectable platelet rich fibrin in combination of bone substitute material for alveolar ridge augmentation - case report". Folia Medica 59 (2017): 362-366.

18. Cortese A., et al. "Platelet-rich fibrin (PRF) in implant dentistry in combination with new bone regenerative technique in elderly patients". International Journal of Surgery Case Reports 28 (2016): 52-56.

19. Mateos L., et al. "Periodontal surgical techniques applied to implantology". Advances in Periodontics 2 (2003): 57-68.

20. Dohan-Ehrenfest DM., et al. "The impact of the centrifuge characteristics and centrifugation protocols on the cells, growth factors and fibrin architecture of a leukocyte- and platelet-rich fibrin (L-PRF) clot and membrane. Part 1: Evaluation of the vibration shocks of 4 models of table centrifuges for L-PRF". Possessed 2 (2014): 129-139.

21. Choukroun J., et al. "Une opportunité in paro-implantology: thePRF”. Implantodontie 42 (2001): 55-62.

22. Giannini S., et al. "Comparison between PRP, PRGF and PRF: Lights and shadows in three similar but different protocols". European Review for Medical and Pharmacological Sciences 19 (2015): 92730. 
23. Khorshidi H., et al. "Comparison of the mechanical properties of early leukocyte-and platelet-rich fibrin versus PRGF / endoret membranes". International Journal of Dentistry (2016): 1849207.

24. Del Corso M., et al. "Current knowledge and perspectives for the use of platelet-rich plasma (PRP) and platelet-rich fibrin (PRF).

25. Solomiya K., et al. "Combination of an allogenic and a xenogenic bone substitue material with injectable platelet-rich fibrin- A comparative in vitro study". The Journal of Biomaterials Applications (2020).

26. De Almeida Barros Mourão CF., et al. "The use of leukocyteand platelet-richfibrin in the management of soft tissue healing and pain in post-extraction sockets: A randomized clinical trial". Journal of Cranio-Maxillofacial Surgery (2020).

27. Law B., et al. "Autogenous free fat graft combined with plateletrich fibrin heals a refractory mandibular osteoradionecrosis". Clinical Therapeutics 171.2 (2020): e110-e113.

28. Fan Y., et al. "Clinical Uses of Platelet-Rich Fibrin in Oral and Maxillofacial Surgery". Dental Clinics of North America 64.2 (2020): 291-303.

29. Bi J., et al. "Platelet-rich Fibrin Improves the Osteo-/Odontogenic Differentiation of Stem Cells from Apical Papilla via the Extracellular Signal-regulated Protein Kinase Signaling Pathway". The Journal of Endodontics (2020).

30. Temmerman A., et al. "Theuse of Leucocyte and platelet-rich fibrin in socket management and ridge preservation: a split mouth, randomized, controlled clinical trial". Journal of Clinic Periodontology (2016).

31. De Angelis P., et al. "Hard and Soft Tissue Evaluation of Different Socket Preservation Procedures Using Leukocyte and Platelet-Rich Fibrin: A Retrospective Clinical and Volumetric Analysis". Journal of Oral and Maxillofacial Surgery 77.9 (2019): 1807-1815.

32. P Dragonas., et al. "Effects of leukocyte-platelet-rich fibrin (L-PRF) in different intraoral bone grafting procedures: a systematic review". International Journal of Oral and Maxillofacial Surgery 48.2 (2019): 250-262.

33. Bielecki T., et al. "The Role of Leukocytes from L-PRP/L-PRF in Wound Healing and Immune Defense: New Perspectives". Current Pharmaceutical Biotechnology 13 (2012): 1153.
34. Pan J., et al. "Effect of platelet-rich fibrin on alveolar ridge preservation". Journal of the American Dental Association 150.9 (2019): 766-778.

\section{Volume 5 Issue 9 September 2021} (C) All rights are reserved by Quevedo Bisonni M., et al. 